\title{
PHASE NOISE AMPLITUDE DEPENDENCE IN SELF-LIMITING WINE-GLASS DISK OSCILLATORS
}

\author{
Seungbae Lee and Clark T.-C. Nguyen \\ Center for Wireless Integrated Microsystems(WIMS) \\ Department of Electrical Engineering and Computer Science \\ University Of Michigan Ann Arbor, Michigan 48109-2122, U.S.A. \\ Tel.:(734)764-3352, Fax:(734)647-1781, email:seungbae@umich.edu
}

\begin{abstract}
Self-limiting 46.4-MHz series resonant reference oscillators employing a wine-glass mode disk micromechanical resonator to avoid hard limiting without the need for ALC have achieved measured phase noises of -113 and $-134 \mathrm{dBc} / \mathrm{Hz}$ at $1 \mathrm{kHz}$ and far-fromcarrier offsets, respectively. When divided down to $10 \mathrm{MHz}$ frequency for fair comparison with other oscillators, these values correspond to $-126 \mathrm{dBc} / \mathrm{Hz}$ and $-147 \mathrm{dBc} / \mathrm{Hz}$, which now satisfy specifications for low-end cellular handsets. For these oscillators, $1 / f^{3}$ phase noise is found to be independent of vibration amplitude for amplitudes below critical Duffing, which pares down the list of possible mechanisms to those that are amplitude independent.
\end{abstract}

\section{INTRODUCTION}

Although their exceptional $Q(>10,000)$ and superb thermal and aging stability make quartz crystals the resonators of choice for precision reference oscillators in portable applications [1], their size and incompatibility with integrated circuits preclude true system-on-a-chip wireless solutions. On the other hand, electrostatically transduced vibrating micromechanical (" $\mu$ mechanical") resonators have become increasingly attractive as frequency-setting elements in reference oscillators for communication applications due to their potential for direct integration or bonded merging with transistors onto single chips [2]-[5]. In particular, phase noise performance nearing the requirements of the GSM cellular telephone standard have recently been demonstrated via an oscillator referenced to an extensional-mode single-crystal silicon vibrating bar with a $Q$ of 200,000 , constructed using a thick-device-layer SOI process [6]. Although the resonator used in [6] lacked adequate temperature stability, another resonator based on surface micromachined polysilicon structural material was recently demonstrated with a temperature coefficient as low as $-0.24 \mathrm{ppm} /{ }^{\circ} \mathrm{C}$ [7], a $Q$ of 4,000 at $10 \mathrm{MHz}$, and a proven integrability with transistor electronics [2][5]. However, an oscillator based on this polysilicon resonator exhibited a $1 / f^{3}$ phase noise component farther from the carrier than expected that prevented the oscillator from achieving GSM-like phase noise specifications (e.g., for $10 \mathrm{MHz}$ reference oscillator, better than $-120 \mathrm{dBc} / \mathrm{Hz}$ at $1 \mathrm{kHz}$ offset frequency, -130 $\mathrm{dBc} / \mathrm{Hz}$ preferred) [8].

Recently, automatic level control (ALC) circuitry designed to limit the vibration amplitude of a micromechanical resonator in an oscillator has been found to successfully remove this $1 / f^{3}$ phase noise [9], suggesting resonator transducer or mechanical nonlinearity as the source of this noise. Unfortunately, ALC removes $1 / f^{3}$ noise at the price of carrier power, so the phase noise performance of ALC'ed oscillators still falls well below the needs of today's cellular wireless standards [9]. In addition, although a mechanism

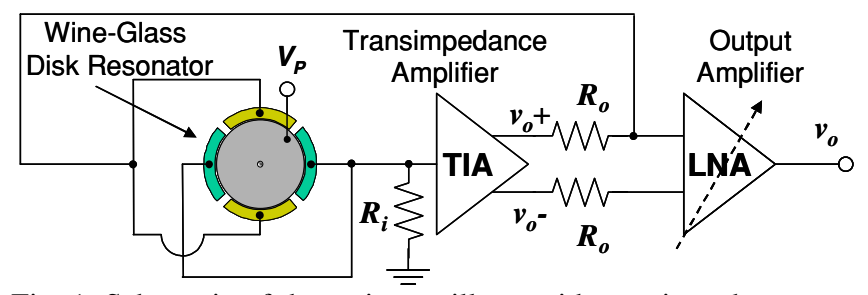

Fig. 1: Schematic of the series oscillator with transimpedance sustaining amplifier utilizing a wine-glass mode disk $\mu$ resonator tank.

linked to resonator amplitude has been identified, an analytical model that accurately predicts the measured $1 / f^{3}$ component has so far been elusive.

Pursuant to both better understanding the source of $1 / f^{3}$ noise and achieving better overall phase noise performance, this work harnesses a recently demonstrated polysilicon wine-glass mode vibrating disk $\mu$ resonator with high- $Q$ even in air [10], and with higher power handling ability (i.e., linearity) than previous surfacemicromachined resonators, to attain oscillators that self-limit before critical point Duffing nonlinearity without the need for ALC. By avoiding hard nonlinearity, these oscillators achieve $10-\mathrm{MHz}-$ effective phase noises of $-123 \mathrm{dBc} / \mathrm{Hz}$ at $1 \mathrm{kHz}$ offset and -147 $\mathrm{dBc} / \mathrm{Hz}$ far-from-carrier under atmospheric pressure, which now satisfy specifications for some cellular handsets. Equally importantly, these oscillators also provide better insight into the amplitude dependence (or independence) of $1 / f^{3}$ phase noise.

\section{OSCILLATOR TOPOLOGY AND OPERATION}

Like a previous oscillator design [9], the oscillator of this work uses a series resonant topology, shown in Fig. 1, in which the wine-glass disk is embedded in a positive feedback loop together with an off-chip NE5211 transresistance sustaining amplifier possessing sufficient gain to initiate and sustain oscillation. As shown, since the micromechanical resonator used for this work is a multiport device (as opposed to the one port devices used in previous work [8][9]), the dc-bias voltage $V_{P}$ is directly connected to the resonator structure without the need for the bias tee used in [8][9]. Other than this and the use of a more advanced micromechanical resonator, the criteria governing start-up and sustenance of oscillation are identical to those described in [9]:

1. For Start-Up: $R_{a m p}>R_{x}+R_{i}+R_{o}$

2. In Steady-State: $R_{\text {amp }}=R_{x}+R_{i}+R_{o}$

3. Loop Phase Condition: $0^{\circ}$ around the positive feedback loop

where $R_{a m p}, R_{i}$, and $R_{o}$ are the gain, input resistance, and output resistance, of the transresistance sustaining amplifier, respectively; and $R_{x}$ is the series motional resistance of the wine-glass disk. As

Travel Support has been generously provided by the Transducers Research Foundation and by the DARPA MEMS and DARPA BioFlips programs. 


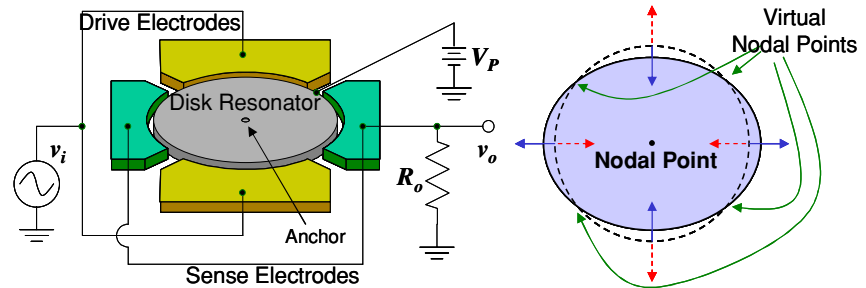

Fig. 2: (a) Perspective-view of a wine-glass disk resonator illustrating a typical two-port bias and excitation scheme, and (b) its mode shape.

will be described, this oscillator limits when its resonator $R_{x}$ increases with amplitude to the point of satisfying criterion 2 above.

\section{RESONATOR AMPLITUDE LIMITATIONS}

Fig. 2(a) presents a more detailed schematic of the disk resonator with electrodes configured to excite the fundamental wine-glass mode shape shown in Fig. 2(b). The dc-bias voltage $V_{P}$ applied to the disk effectively amplifies the force and motional output current induced when a resonance ac excitation voltage $v_{i}$ forces the disk into resonance vibration. The motion is constrained entirely to the plane of the disk, with its perimeter expanding along one axis and contracting in the orthogonal axis about the center point, which remains stationary, acting as a node. Although four additional quasi-nodal points exist on the disk perimeter and thereby allow greater flexibility in the choice of support structure [10], this work simply anchors the disk at its center in order to maximize the available electrode area. The wine-glass resonance frequency $f_{o}$, goes as the inverse of its radius $R$, and can be obtained by solving the mode frequency equation given in [10]

At the amplitudes experienced during oscillator vibration, the force $F$ needed to achieve a given vibration amplitude $x$ for this electrostatically driven resonator becomes of a function of thirdorder Duffing nonlinearity [11] and is governed by the expression

$$
\frac{F}{m_{r}}=\ddot{x}+\frac{\omega_{o}}{Q} \dot{x}+\frac{k_{1}}{m_{r}} x-\frac{k_{3}}{m_{r}} x^{3}=\ddot{x}+\frac{\omega_{o}}{Q} \dot{x}+\omega_{o}^{2}\left(x-\mu x^{3}\right)
$$

where, $k_{1}=k_{m 1}-k_{e 1}, \quad k_{3}=k_{e 3}-k_{m 3}, \quad \omega_{o}=\sqrt{\frac{k_{1}}{m_{r}}}, \quad \mu=\frac{k_{3}}{k_{1}}$,

and where $m_{r}$ is effective mass of resonator; $k_{m 1}$ and $k_{e 1}$ are the linear mechanical and electrical spring constants, respectively; and $k_{m 3}$ and $k_{e 3}$ model their third-order spring nonlinearities. For the resonators of this work, the electrode-to-resonator gaps $d_{\mathrm{o}}$ are quite small, so $k_{e 3}>k_{m 3}$ (i.e., the third-order coefficient in (1) is negative), so spring softening occurs as the vibration amplitude increases. The resonance frequency then decreases as the resonator amplitude increases, resulting in a frequency response curve that bends leftward as amplitudes increase, as illustrated in Fig. 3, where the amplitude dependence of the peak (radian) frequency $\omega$ is governed by the expression [11]

$$
\omega^{2}=\omega_{o}^{2}-\frac{3}{4} \frac{k_{3}}{m_{r}} x^{2}
$$

where $\omega_{o}$ is the radian resonance frequency at small amplitudes.

When the peak vibration amplitude exceeds the critical point:

$$
X_{c r i t} \approx \frac{1.52}{\sqrt{\mu Q}}
$$

the amplitude versus frequency curve bends to a point where there

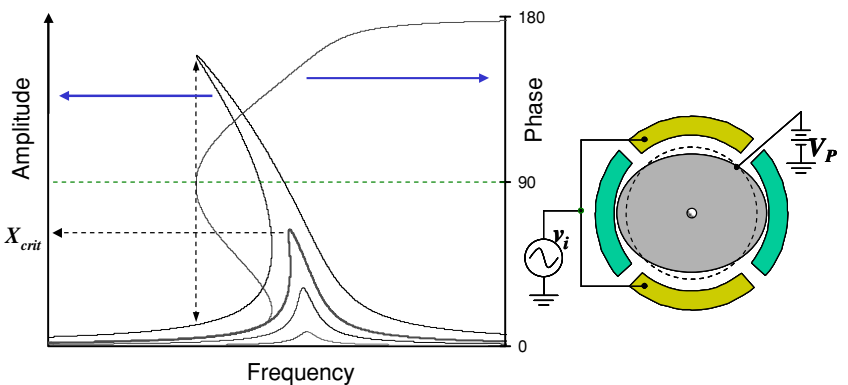

Fig. 3: Schematic depiction of the theoretical Duffing curve expected for large amplitudes.

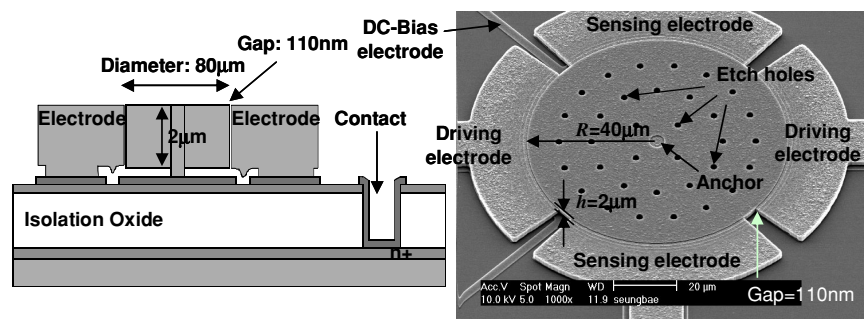

Fig. 4: Cross section and SEM of a 46.4-MHz wine-glass mode disk polysilicon $\mu$ resonator, with dimensions indicated.

are three theoretically valid amplitudes for each frequency in the bent portion of the curve. This amplitude ambiguity leads to unstable steady-state operation [2], which then results in excessive phase noise. Needless to say, operation at amplitudes past the critical point are not recommended, so $X_{\text {crit }}$ effectively defines an amplitude threshold that then sets the maximum rms power handling ability of the resonator to

$$
P_{o}=\frac{1}{2} \frac{\omega_{o} k_{1}}{Q} x^{2} \leq \frac{1}{2} \frac{\omega_{o} k_{1}}{Q} X_{c r i t}^{2}=1.16 \frac{\omega_{o} k_{1}}{\mu Q^{2}}
$$

\section{EXPERIMENTAL RESULTS}

46.4-MHz polysilicon wine-glass mode disk resonators were designed and fabricated using a process similar to a previously reported self-aligned stem process [12], except for the use of a chemical mechanical polishing (CMP) step to reduce topography at the end of the process. Fig. 4 presents the final cross section and scanning electron micrograph (SEM) of one such resonator, identifying key features and dimensions. With $80-\mu \mathrm{m}$ diameters, the disks of this work are larger than previous ones, so etch holes are now used to keep release etch times under 15 minutes. This is needed to prevent HF attack of the nitride isolation layer underneath the devices, which occurs when the nitride is exposed to 49 wt.\% HF for longer than 35 minutes. Structurally, the etch holes reduce the resonator stiffness, resulting in a slight reduction of its resonance frequency. Finite element simulation using ANSYS predicts frequencies of $46.7-\mathrm{MHz}$ and $48.1-\mathrm{MHz}$ for wine-glass mode disk resonators with and without etch holes, respectively.

Fig. 5 presents frequency spectra measured for the device of Fig. 4 under 50 mTorr pressure in a custom-built vacuum chamber, and in air as well, where the dependence of frequency on dc-biasderived electrical stiffness [13] and on increased gas damping in air can be clearly seen. In vacuum, the $Q$ is 30,267. In air, although a larger dc-bias $V_{P}$ is needed to achieve the same motional resistance, the $Q$ is still a respectable 5,091. The oscillator circuit of Fig. 1 was implemented on a printed circuit (pc) board shaped to allow placement within the custom-built vacuum chamber. Dies containing fabricated wine-glass mode disk $\mu$ resonators were glued to this 


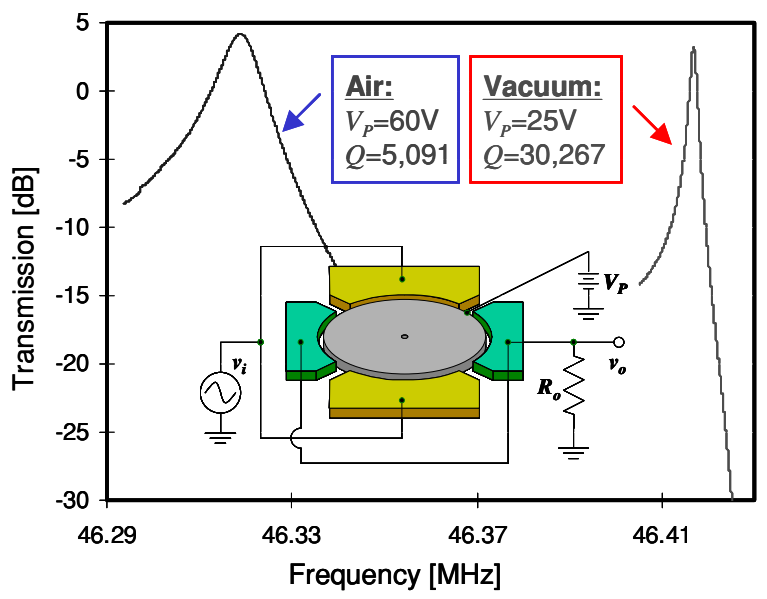

Fig. 5: Measured frequency characteristics for the wine-glass mode disk $\mu$ resonator in air as well as in vacuum, with an inset specifying the measurement setup.
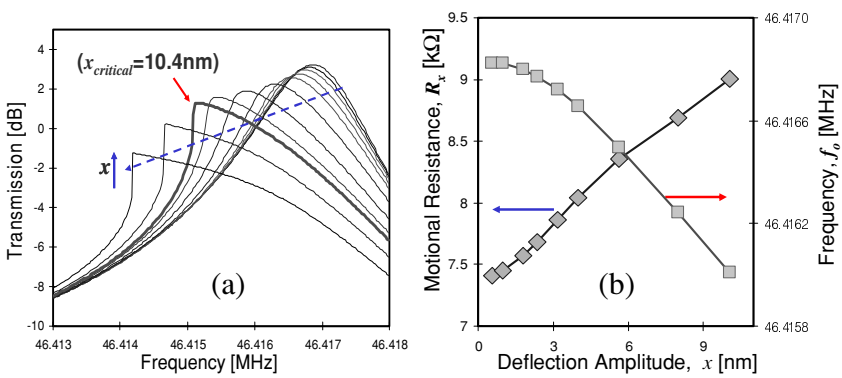

Fig. 6: Measured plots summarizing the displacement amplitude dependence of the wine-glass disk resonator: (a) frequency responses, showing Duffing nonlinearity; and (b) motional resistance $R_{x}$ and resonance frequency; all measured under 50 mTorr vacuum.

board over specially grounded regions and bonded to boardmounted electronics.

Fig. 6(a) presents measured plots of transmission versus frequency for a $46.4-\mathrm{MHz}$ wine-glass mode disk under increasing drive force amplitudes, where spring softening Duffing behavior is clearly seen. In particular, the peak frequency clearly decreases with increasing displacement amplitude. In addition, a decrease in peak transmission is also seen, consistent with [2], where either $Q$ or transducer electromechanical coupling, or both, may be decreasing with displacement amplitude. This reduction in transmission corresponds to an increase in series motional resistance $R_{x}$ with displacement amplitude, plotted in Fig. 6(b) (along with frequency), which then provides a convenient mechanism for self-limiting of an oscillator referenced to a wine-glass mode micro-disk. In particular, at oscillator start-up, when the $R_{x}$ of the resonator is smaller than the transresistance gain of the sustaining amplifier $R_{a m p}$, the overall loop gain is greater than unity, so oscillations build up. As the amplitude of the resonator grows, however, $R_{x}$ grows until it equals $R_{\text {amp }}$ minus other series loop resistances (e.g., amplifier input and output resistance), at which point, the oscillation amplitude stops growing, and steady-state operation is achieved. In this mode of operation, the steady-state oscillation amplitude can be set by setting the initial difference between $R_{x}$ and $R_{a m p}$, which can in turn be set by choice of dc-bias voltage $V_{P}$ [9]. The wine-glass disk of this work has an advantage over other resonators in that its power handling and $R_{x}$ versus amplitude characteristic are such that oscillation with sufficient output power can be achieved at vibration amplitudes well below critical Duffing, which is one of

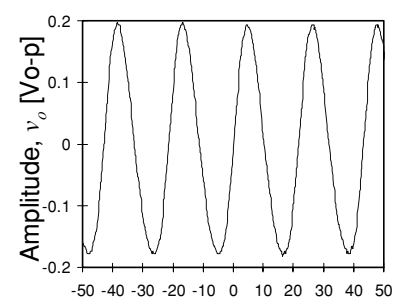

(a) time [ns]

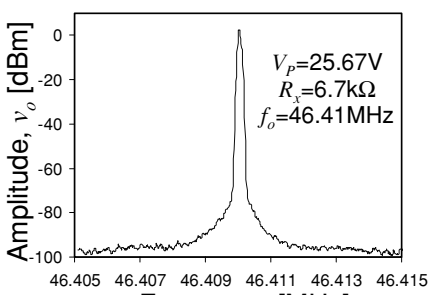

(b) Frequency $[\mathrm{MHz}$
Fig. 7: Measured output (a) waveform and (b) Fourier spectrum for the self-limiting wine-glass disk micromechanical oscillator.
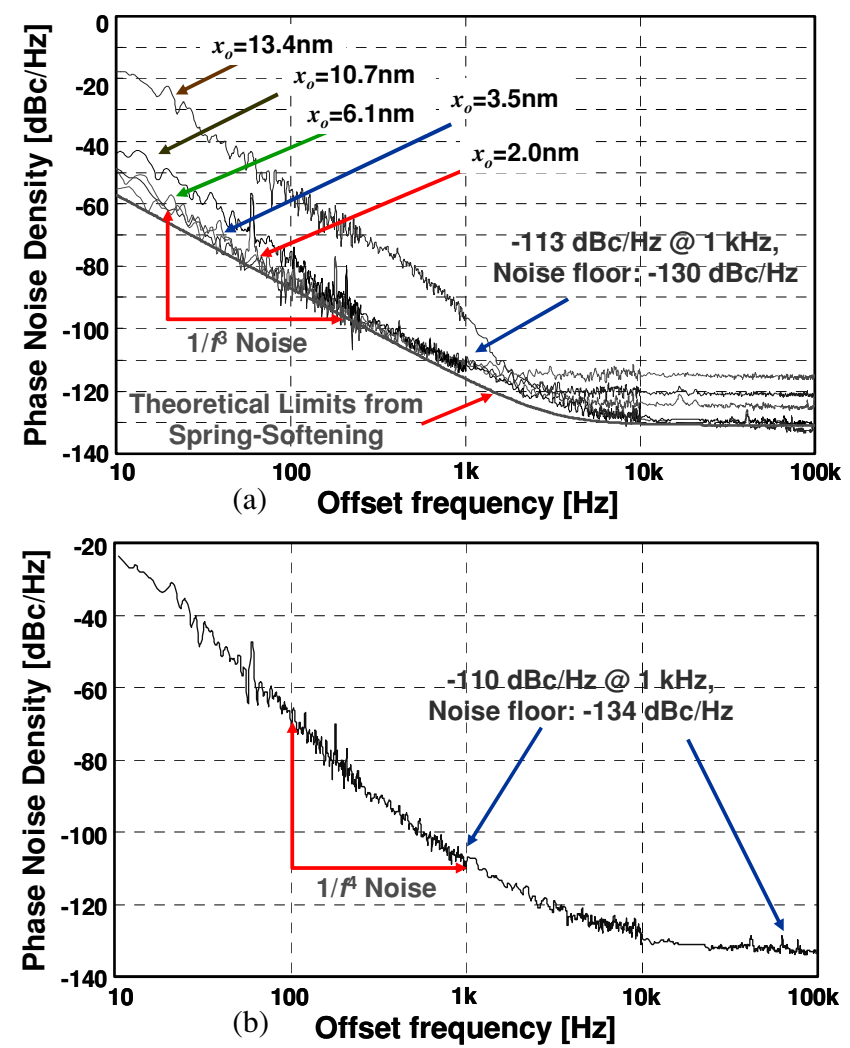

Fig. 8: Measured phase noise density-to-carrier power ratio versus carrier offset frequency for the $\mu$ mechanical resonator oscillator in (a) vacuum and (b) in atmosphere.

the criteria for good oscillator stability in Section III.

Fig. 7 presents the measured output waveform and Fourier spectrum for a self-limiting wine-glass disk oscillator hooked up as in Fig. 1 and operated under conditions summarized in the inset. Fig. 8 presents plots of phase noise density versus frequency offset for the 46.4-MHz wine-glass disk oscillator, with displacement amplitude as a third variable, measured (a) under 50 mTorr vacuum and (b) in air, using an HP E5500 Phase Noise Measurement System. Operational specifics for each curve are summarized in Table I. In vacuum, a $1 / f^{3}$ component is seen close to the carrier, and this noise clearly depends upon the oscillation amplitude, becoming considerably worse when the amplitude of oscillation becomes larger than the critical Duffing amplitude $(10.4 \mathrm{~nm})$, where multiple operating amplitudes are possible in the curves of Fig. 6(a), verifying that operation past the critical point should be avoided.

In vacuum, the phase noise at $1 \mathrm{kHz}$ offset from the carrier is of the $1 / f^{3}$ variety, as expected, but curiously it remains fairly constant at $-113 \mathrm{dBc} / \mathrm{Hz}$ for amplitudes below the critical amplitude. On the other hand, the far-from-carrier phase noise has a rather strong 
Table I. Operational Data for Various Oscillation Amplitudes

\begin{tabular}{|c||c|c|c|c|c|}
\hline $\begin{array}{c}\text { Amplitude, } \\
\boldsymbol{x}[\mathbf{n m}]\end{array}$ & $\mathbf{2 . 0}$ & $\mathbf{3 . 5}$ & $\mathbf{6 . 1}$ & $\mathbf{1 0 . 7}$ & $\mathbf{1 3 . 4}$ \\
\hline \hline $\boldsymbol{V}_{\boldsymbol{P}}[\mathbf{V}]$ & 24.54 & 24.67 & 24.93 & 25.67 & 26.96 \\
\hline $\boldsymbol{R}_{\boldsymbol{x}}[\mathrm{k} \boldsymbol{\Omega}]$ & 7.323 & 7.245 & 7.095 & 6.691 & 6.066 \\
\hline $\boldsymbol{f}_{\boldsymbol{o}}[\mathbf{M H z}]$ & 46.4131 & 46.4124 & 46.4116 & 46.4093 & 46.3990 \\
\hline $\boldsymbol{v}_{\boldsymbol{i}}[\mathbf{m V}]$ & 34.6 & 61.5 & 109.4 & 194.5 & 259.4 \\
\hline $\boldsymbol{L}\left[\boldsymbol{f}_{\boldsymbol{m}}=\mathbf{1 k H z}\right]$ & -111 & -111 & -112 & -113 & -97 \\
\hline Noise Floor & -115.2 & -121.4 & -125.3 & -130.5 & -132.3 \\
\hline
\end{tabular}

dependence on amplitude, getting smaller with larger displacement amplitude, and attaining its lowest value of $-130 \mathrm{dBc} / \mathrm{Hz}$ at an amplitude of $10.7 \mathrm{~nm}$, right at the edge of critical Duffing. When divided down to $10 \mathrm{MHz}$ for fair comparison with other standard oscillators, the phase noise for the $10.7 \mathrm{~nm}$ displacement case at $1 \mathrm{kHz}$ and far-from-carrier offsets are -126 and $-143 \mathrm{dBc} / \mathrm{Hz}$, respectively, which are now in the range of acceptable values for cellular handsets, although some sticklers might still demand -130 and $-150 \mathrm{dBc} / \mathrm{Hz}$. If needed, these values should be attainable via a redesign to provide a more favorable Duffing response curve (e.g., by optimizing the electrode-to-resonator gap spacing).

In air (Fig. 8(b)), although a mysterious $1 / f^{4}$ phase noise component introduces itself at carrier offset frequencies below $1 \mathrm{kHz}$, the overall performance of the oscillator matches that of the vacuum-operated version past $1 \mathrm{kHz}$, despite its lower $Q$. The performance even exceeds that in vacuum at far-from-carrier offsets, achieving $4 \mathrm{~dB}$ better in this region. The reason: In air the amplitude required to make $R_{x}=R_{\text {amp }}$ is smaller than that needed in vacuum, and the dc-bias voltage $V_{P}$ needed is larger-a combined condition that allows both small displacement amplitude for good close-to-carrier phase noise, but large current output amplitude (provided by the larger $V_{P}$ ) for good far-from-carrier noise.

\section{INSIGHTS INTO $1 / f^{3}$ PHASE NOISE}

The observation that $1 / f^{3}$ phase noise is independent of vibration amplitude for amplitudes below critical Duffing pares down the list of possible mechanisms to those that are amplitude independent. Among these mechanisms is the previously proposed [8] shaping of aliased $1 / f$ noise by the resonator passband, leading to a $1 / f^{3}$ phase noise component governed by

$$
L\left[f_{m}\right]=\left[\frac{1}{4 Q_{l}^{2} V_{P}^{2}}+\frac{1}{4 k_{1}^{2}} \frac{\left(\varepsilon_{o} A_{o}\right)^{2}}{d_{o}^{6}} V_{P}^{2}\right] \cdot 2 q K_{1} I_{B} R_{a m p}^{2} \frac{f_{o}^{2}}{f_{m}^{3}}
$$

where $d_{o}$ and $A_{o}$ are the electrode-to-resonator gap and overlap area, respectively; and $K_{1}(50 \mathrm{kHz})$ and $I_{B}(50 \mu \mathrm{A})$ are the $1 / f$ noise constant and base current, respectively, of the bipolar transistor at the input of the sustaining transimpedance amplifier with gain $R_{\text {amp }}, 14$ $\mathrm{k} \Omega$.

Another possible mechanism arises when $1 / f$ noise on the dcbias voltage $V_{P}$ leads to electrical-stiffness-induced frequency instability, which then generates a $1 / f^{3}$ phase noise component governed by

$$
L\left[f_{m}\right] \approx \frac{1}{2}\left(\frac{\varepsilon_{o} A_{o} V_{P}}{k_{m 1} d_{o}^{3}}\right)^{2} 2 q K_{1} I_{B} R_{a m p}^{2} \frac{f_{o}^{2}}{f_{m}^{3}}
$$

Unfortunately, neither of the above equations exactly matches the measured phase noise of Fig. 8. In particular, using the data in the $4^{\text {th }}$ column of Table I, (6) predicts $-124 \mathrm{dBc} / \mathrm{Hz}$ at $1 \mathrm{kHz}$ offset, while (7) yields $-120 \mathrm{dBc} / \mathrm{Hz}$ - each more than $-7 \mathrm{dBc} / \mathrm{Hz}$ from the measured value. A new formulation based on frequency shifts due to Duffing distortion (3) leads to the expression

$$
L\left[f_{m}\right] \approx \frac{1}{(2 \pi)^{4} f_{o}^{2}} \cdot\left(\frac{3 k_{3}}{4 m_{r}}\right)^{2} \frac{4 Q^{2} V_{P}^{2}}{k_{1}^{2}} \cdot \frac{\left(\varepsilon_{o} A_{o}\right)^{2}}{d_{o}^{6}} \cdot 2 q K_{1} I_{B} R_{S}^{2} \cdot \frac{x^{4}}{f_{m}^{3}}
$$

This expression yields $-115 \mathrm{dBc} / \mathrm{Hz}$ at $1 \mathrm{kHz}$ using the data in the $4^{\text {th }}$ column of Table I, which is very close to the measured value. However, this expression also exhibits a dependence on amplitude not seen in measurement, so cannot be entirely correct.

Perhaps the previous observation that ALC or limiting via electronics rather than the resonator eliminates $1 / f^{3}$ phase noise is more telling. In particular, the mechanism for $1 / f^{3}$ phase noise is likely tied to the Duffing distortion-based limiting mechanism described in Section III. Efforts to properly model $1 / f^{3}$ phase noise are ongoing.

\section{CONCLUSIONS}

A 46.4-MHz $\mu$ mechanical resonator reference oscillator has been demonstrated using a series resonant oscillator topology in which a transimpedance amplifier with zero phase shift sustains the oscillation of a high stiffness wine-glass mode $\mu$ mechanical disk resonator. Due to the high- $Q$, high power handling, and large stiffness (relative to previous micromechanical devices), of the wine-glass disk resonator, this oscillator achieves a phase noise performance consistent with the needs of commercial wireless communications without the need for ALC. This performance is attained as long as the vibration amplitude of the resonator is kept below the critical Duffing amplitude; otherwise, excessive phase noise ensues. In addition, without ALC or some electronic means for limiting, this oscillator still exhibits the $1 / f^{3}$ close-to-carrier phase noise seen in previous micromechanical oscillators, further verifying that limiting via resonator nonlinearity (e.g., Duffingrelated nonlinearity) is likely responsible for this noise. The fact that $1 / f^{3}$ noise is found to be independent of vibration amplitude for operation below critical Duffing sheds more light on the roots of this noise phenomenon.

Acknowledgment: This work was supported under DARPA Cooperative Agmt. No. F30602-01-1-0573.

References:

[1] R. J. Matthys, Crystal Oscillator Circuits. New York: Wiley, 1983.

[2] C. T.-C. Nguyen, et al., IEEE J. Solid-State Circuits, vol. 34, no. 4, pp. 440-455, April 1999.

[3] T. A. Core, et al., Solid State Technology, pp. 39-47, Oct. 1993.

[4] A. E. Franke, et al., Tech. Digest, $12^{\text {th }}$ Int. IEEE MEMS Conf., Orlando, Florida, Jan. 17-21, 1999, pp. 630-637.

[5] A.-C. Wong, et al., Dig. of Tech. Papers, Transducers'01, Munich, Germany, June 10-14, 2001, pp. 992-995.

[6] T. Mattila, et al., Sensors and Actuators, A 101, pp. 1-9, 2002.

[7] W. -T. Hsu, et al., Tech. Digest, 2002 IEEE Int. Micro Electro Mechanical Systems Conf., Jan. 20-24, 2002, pp. 731-734.

[8] S. Lee, et al., Digest of Technical Papers, Transducers'01, Munich, Germany, June 10-14, 2001, pp. 1094-1097.

[9] S. Lee, et al., Proceedings of 2003 IEEE Frequency Control Symposium, Tampa, Florida, May 5-8, 2003, pp.341-349.

[10] M. A. Abdelmoneum, et al., Technical Digest, $16^{\text {th }}$ Int. IEEE Micro Electro Mechanical Systems Conf., 2003, pp. 698-701.

[11] S. Timoshenko, D. H. Young, and W. Weaver Jr., Vibration Problems in Engineering. $5^{\text {th }}$ ed. New York: Wiley, 1990.

[12] J. Wang, et al., Dig. of Tech. Papers, Transducers'03, 2003, pp. 947-950.

[13] F. D. Bannon III, et al., IEEE J. Solid-State Circuits, vol. 35, no. 4, pp. 512-526, April 2000. 